PoS $\quad \begin{aligned} & \text { PROCEEDINGS } \\ & \text { OF SCIENCE }\end{aligned}$

\title{
Methanol maser polarization probing magnetic fields during high-mass star formation
}

\section{Wouter Vlemmings*}

Argelander Institute for Astronomy, University of Bonn, Germany

E-mail: wouter@astro.uni-bonn.de

\begin{abstract}
Recent observations have shown methanol masers to be excellent probes of the magnetic field in high-mass starforming regions. In addition to the first maps of linearly polarized $6.7 \mathrm{GHz}$ methanol masers, circular polarization has been detected on a large sample of methanol sources with the Effelsberg telescope. Here I present on overview of a number of projects aimed to reveal the strength and structure of magnetic fields in massive starforming regions.
\end{abstract}

The 9th European VLBI Network Symposium on The role of VLBI in the Golden Age for Radio Astronomy and EVN Users Meeting

September 23-26, 2008

Bologna, Italy

${ }^{*}$ Speaker. 


\section{Introduction}

Although massive stars play an important role in the chemical and energetic evolution of their host galaxies, their formation mechanism remains elusive. This problem is the topic of extensive observational and theoretical efforts. Even though few of the current simulations include magnetic fields, the influence of magnetism on the star formation processes is extensive as it can support a molecular cloud against collapse, affect core fragmentation and change the feedback processes.

Most current high-mass star formation magnetic field information comes from $\mathrm{H}_{2} \mathrm{O}$ and $\mathrm{OH}$ maser polarization observations [6] (and references therein). The observations of the $\mathrm{H}_{2} \mathrm{O}$ maser Zeeman effect using Very Long Baseline Interferometry (VLBI) reveal field strengths between 10 and $600 \mathrm{mG}$, while the linear polarization measurements reveal a complex but often ordered magnetic field morphology (e.g. [4]). Aside from $\mathrm{H}_{2} \mathrm{O}$ masers, tracing high density regions $\left(n_{\mathrm{H}_{2}} \approx\right.$ $10^{8}-10^{11} \mathrm{~cm}^{-3}$ ), the magnetic field in the less dense surrounding regions is typically probed by polarimetric $\mathrm{OH}$ maser observations (e.g. [1]). These observations reveal fields of a few $\mathrm{mG}$ as well as ordered structure in the magnetic field. However, the strongest and most abundant of the high-mass star formation region masers arises from the $6.7 \mathrm{GHz} 5_{1}-6_{0} A^{+}$methanol transition, and for this maser hitherto only very few polarization observations exist. Like $\mathrm{H}_{2} \mathrm{O}$, methanol is a non-paramagnetic molecule, and thus both the linear and circular polarization fractions are small. The first polarization measurements were made with the Australia Telescope Compact Array (ATCA) on the $6.7 \mathrm{GHz}$ maser toward a handful of southern massive star forming regions and linear polarization between few and 10\% was detected [3]. The first high angular resolution linear polarization maps were recently made using MERLIN [5] and the Long Baseline Array [2]. The MERLIN observations are discussed in more detail in §.2. In addition to the linear polarization observations, observations with the Effelsberg telescope have for the first time revealed the Zeeman splitting of the $6.7 \mathrm{GHz}$ methanol masers [7]. These observations are shortly discussed in §.3.

\section{MERLIN observations}

The $6.7 \mathrm{GHz}$ methanol masers of the UCHII region W3(OH) were observed on December 1011 in 2004 using MERLIN. A $500 \mathrm{kHz}$ bandwidth with 512 spectral channels was used, centered at $V_{\mathrm{LSR}}=-45 \mathrm{~km} / \mathrm{s}$, resulting in a velocity resolution of $0.044 \mathrm{~km} / \mathrm{s}$ and a total velocity coverage of $22.5 \mathrm{~km} / \mathrm{s}$. The details of the calibration are described in [5]. A map of the strongest $6.7 \mathrm{GHz}$ maser features together with their polarization angles is shown in Fig. 1.

The fractional linear polarization of the $6.7 \mathrm{GHz}$ methanol masers is comparable to that found in the Southern star-forming region, NGC6334F, by [3]. For the masers in W3(OH), the error weighted median $\left\langle P_{L}\right\rangle_{\text {med }}=1.8 \pm 0.7 \%$, while the maximum linear polarization fraction is $\sim 8 \%$. We find that, as seen in Fig. 1, the polarization angles in $\mathrm{W} 3(\mathrm{OH})$ are similar across the entire maser region with the exception of those in part of the complex region (near $\Delta \alpha, \Delta \delta=0.3,0.7 \operatorname{arcsec}$ ). The error weighted median of the polarization $\langle\chi\rangle_{\text {med }}=-67 \pm 9^{\circ}$. In Fig. 1 we have also indicated the polarization vectors of the ground-state $\mathrm{OH}$ masers at $1.6 \mathrm{GHz}$ from the electronic tables of [8], for which linear polarization has been detected at more than a $5 \sigma$ level. The $\mathrm{OH}$ maser polarization angles show a wider spread, with the median polarization angle $\langle\chi\rangle_{\text {medOH }_{\mathrm{OH}}}=104 \pm 27^{\circ}$, which, for the $1.6 \mathrm{GHz} \mathrm{OH}$ masers, has been attributed to Faraday rotation along the maser path. Faraday 


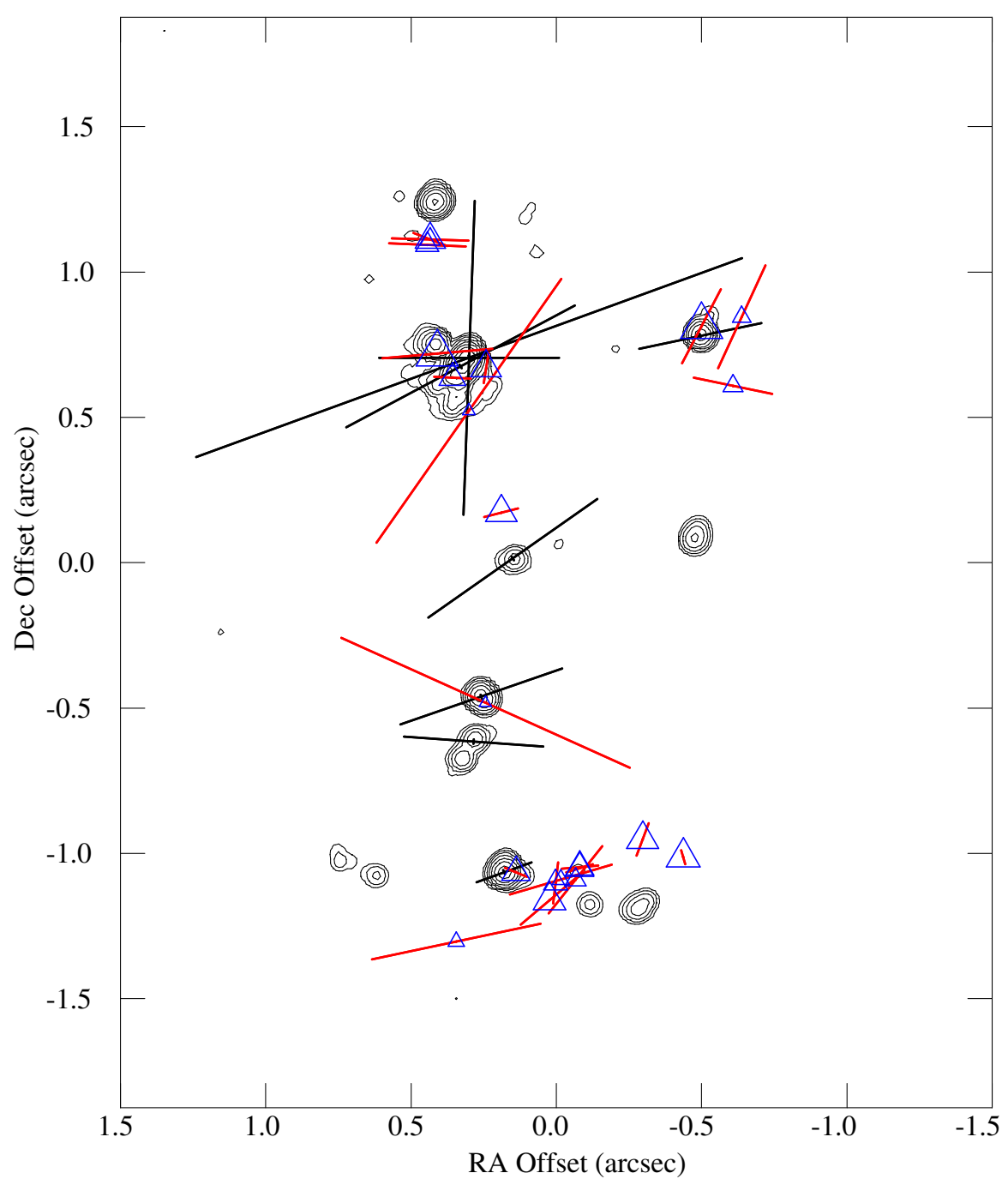

Figure 1: The methanol masers of $\mathrm{W} 3(\mathrm{OH})$ (contours) including the polarization vectors (black) scaled linearly according the fractional linear polarization $\left(P_{L}\right)$. The blue triangles denote the main line $\mathrm{OH}$ masers from [8] for which polarized intensity was detected at $5 \sigma$ significance (polarized flu $x>75 \mathrm{mJy}$ ), and the red vectors are their linearly scaled polarization vectors. The main line $\mathrm{OH}$ maser polarization vectors lengths are scaled down by a facto $r$ of 5 with respect to the lengths of the methanol maser polarization vectors. 
rotation can be described by:

$$
\Phi\left[^{\circ}\right]=4.17 \times 10^{6} D[\mathrm{kpc}] n_{e}\left[\mathrm{~cm}^{-3}\right] B_{\|}[\mathrm{mG}] v^{-2}[\mathrm{GHz}]
$$

where $D$ is the length of the path over which the Faraday rotation occurs, $n_{e}$ and $B_{\|}$are respectively the average electron density and magnetic field along this path and $v$ is the frequency.For typical values of the interstellar electron density $n_{e} \approx 0.04 \mathrm{~cm}^{-3}$ and magnetic field $B_{\|} \approx 1.5 \mu \mathrm{G}$, and assuming a distance to $\mathrm{W} 3(\mathrm{OH})$ of $D \approx 1.95 \mathrm{kpc}$, the Faraday rotation at $6.7 \mathrm{GHz}$ is $\Phi \approx 11^{\circ}$. The Faraday rotation at $1.6 \mathrm{GHz}$ on the other hand is $\Phi \approx 190^{\circ}$. Thus, the difference between the median polarization angles at $6.7 \mathrm{GHz}$ and $1.6 \mathrm{GHz}, \Delta \chi=171 \pm 28^{\circ}$, is probably solely caused by external Faraday rotation.

\section{Effelsberg observations}

In addition to linear polarization, circular polarization due to Zeeman splitting was recently detected in a large sample of $6.7 \mathrm{GHz}$ methanol masers using the Effelsberg telescope [7]. Where the linear polarization fraction is of order $2 \%$, circular polarization is found to be of order $0.5 \%$. An example of the observed circular polarization is shown in Fig.2.
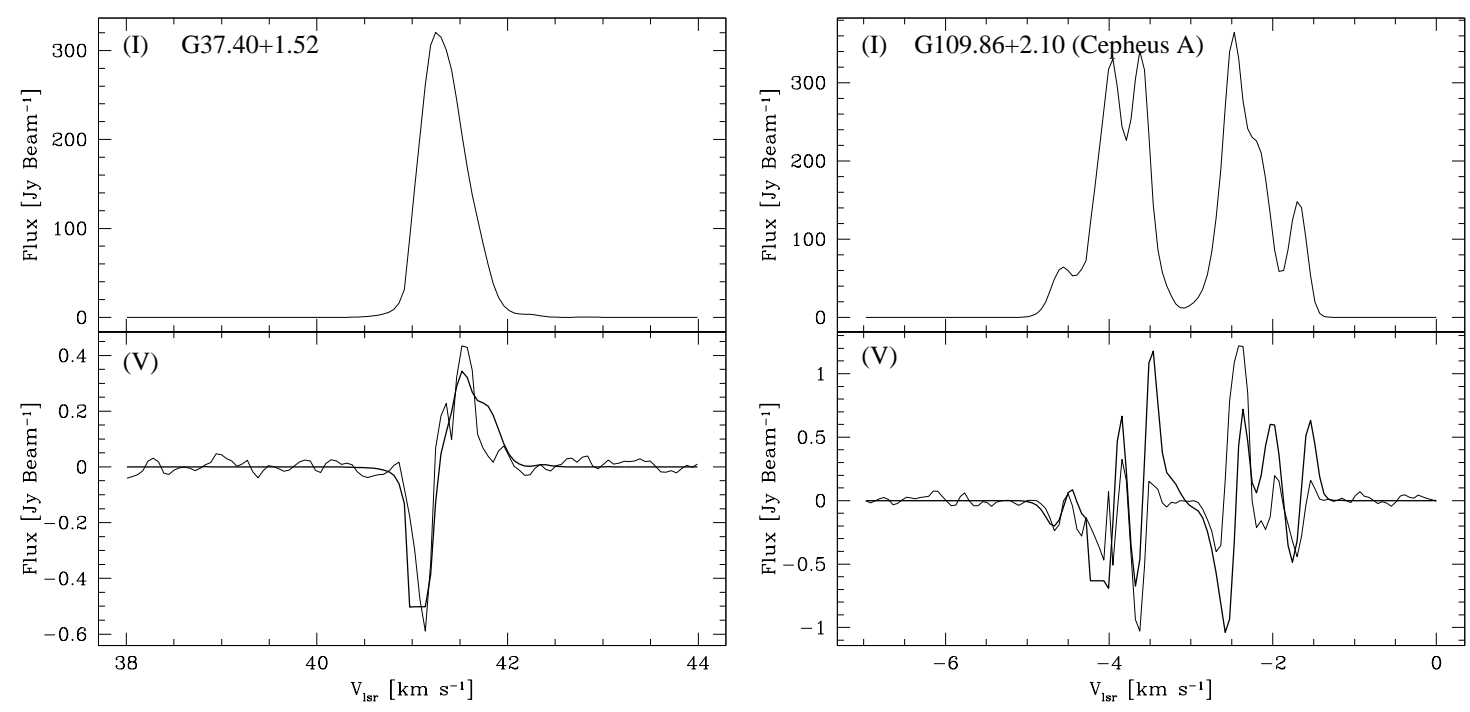

Figure 2: Total intensity and circular polarization spectrum for G37.40+1.52 (left) and G109.86+2.10 (Cepheus A; right). The thick solid line in the bottom panel is best fit fractional total power derivative to the circular polarization spectrum

Significant Zeeman splitting was detected for 17 out of 24 sources and indicates an absolute magnetic field strength component $\left|B_{\|}\right|$along the maser propagation direction between 2.8 and $42 \mathrm{mG}$ (Fig.3. Weighing the field strength by measurement significance, the average magnetic field $\left\langle B_{\|, \text {meth }}\right\rangle=12 \mathrm{mG}$. This needs to be corrected for a random angle between magnetic field and 


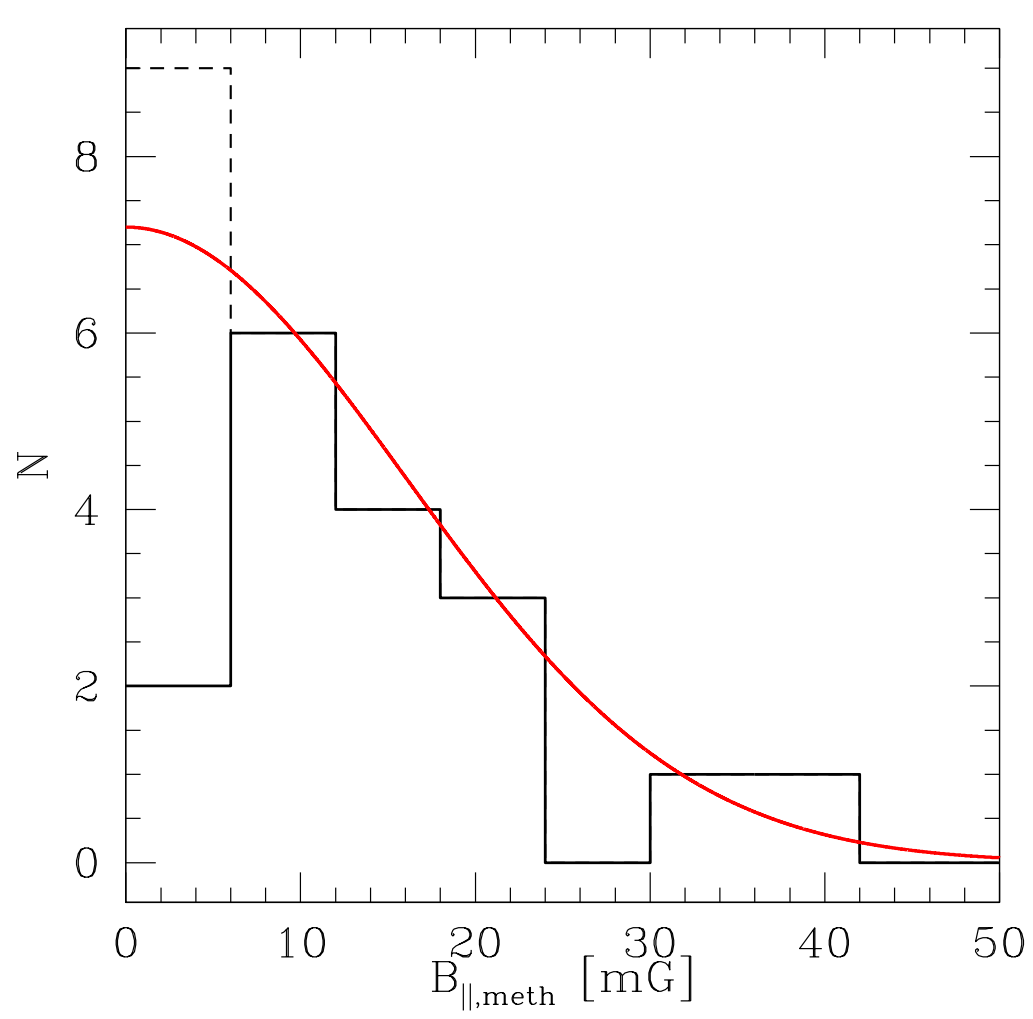

Figure 3: Distribution of the line-of-sight magnetic field strengths $B_{\|}$determined from Effelsberg $6.7 \mathrm{GHz}$ methanol maser Zeeman measurements. The dashed line includes the non-detection while the solid curve indicates the distribution expected from a randomly oriented magnetic field with $B_{\|}=12 \mathrm{mG}$.

the line-of-sight, which implies for the absolute field strength $|B|=2\left\langle B_{\|}\right\rangle$. This gives $\left|B_{\text {meth }}\right|=$ $23 \pm 6 \mathrm{mG}$. The error on the absolute field strength is dominated by the estimated uncertainty in the Zeeman coefficient. This field strength is larger than that found in the $\mathrm{OH}$ maser regions, with the average $\mathrm{OH}$ maser determined field strength being $\sim 4 \mathrm{mG}$.

The dynamical importance of the magnetic field can be quantified by defining a critical magnetic field strength $B_{\text {crit }}=\left(8 \pi \rho v^{2}\right)^{1 / 2}$ for which the dynamic and magnetic pressure are equal. Here $\rho$ and $v$ are the density and velocity of the maser medium respectively. The current observational limits suggest that the majority of the $6.7 \mathrm{GHz}$ maser sources occur near the high-density limit of the maser range $\left(n_{\mathrm{H}_{2}}=10^{7}-10^{9} \mathrm{~cm}^{-3}\right)$, with a typical value of $n_{\mathrm{H}_{2}}=10^{8} \mathrm{~cm}^{-3}$. Taking this density and a typical gas velocity of $\lesssim 5 \mathrm{~km} / \mathrm{s}, B_{\text {crit }} \approx 12 \mathrm{mG}$. The measured magnetic field vales are thus comparable to $B_{\text {crit }}$ and hence dynamically important.

\section{Summary \& Outlook}

Methanol masers have been shown to be able to probe magnetic field strength and structure in dense regions of massive starformation. In $\mathrm{W} 3(\mathrm{OH})$, a large difference is found between methanol 
and $\mathrm{OH}$ maser polarization angles due to external Faraday rotation. This highlights the limits of $\mathrm{OH}$ maser polarimetry in studying the intrinsic large scale structure of magnetic fields. As methanol masers are much less affected by Faraday rotation, they are a more promising tool to study magnetic field morphology, despite their much lower fractional linear polarization of $\sim 2 \%$. Using the Effelsberg 100-m telescope, the first Zeeman splitting observations were made in a sample of 24 massive starforming regions, indicating a magnetic field strength of $\left|B_{\text {meth }}\right|=23 \pm 6 \mathrm{mG}$.

A number of projects exploiting the exciting possibilities of methanol masers in starformation magnetic field research have been initiated. We have greatly expanded the sample of Zeeman splitting measurements using the Parkes telescope to include all strong Southern hemisphere $6.7 \mathrm{GHz}$ methanol masers. Recent observations using the EVN will be used in an attempt to map the magnetic field of $\mathrm{W} 75 \mathrm{~N}$ at the highest angular resolution and MERLIN observations of Cepheus A reveal an ordered magnetic field along an ellipsoidal maser distribution. eMerlin observations of a large sample of methanol masers in massive starforming regions have been proposed as part of the "Feedback during massive starformation" legacy project to determine the full 3D magnetic field structure close to protostellar outflows.

\section{References}

[1] Bartkiewicz, A., Szymczak, M., Cohen, R. J., \& Richards, A. M. S. 2005, MNRAS, 361, 623

[2] Dodson, R. 2008, A\&A, 480, 767

[3] Ellingsen, S. P. 2002, in IAU Symposium, Vol. 206, Cosmic Masers: From Proto-Stars to Black Holes, ed. V. Migenes \& M. J. Reid, 151

[4] Vlemmings, W. H. T., Diamond, P. J., van Langevelde, H. J., \& Torrelles, J. M. 2006a, A\&A, 448, 597

[5] Vlemmings, W. H. T., Harvey-Smith, L., \& Cohen, R. J. 2006b, MNRAS, 371, L26

[6] Vlemmings, W. H. T. 2007, IAU Symposium, 242, 37

[7] Vlemmings, W. H. T. 2008, A\&A, 484, 773

[8] Wright M. M., Gray M. D., Diamond P. J., 2004, MNRAS, 350, 1253 